\title{
Benefícios da inteligência artificial na identificação de cárie dentária: revisão
}

\section{integrativa}

\author{
Benefits of artificial intelligence in dental caries identification: integrative review
}

Beneficios de la inteligencia artificial en la identificación de caries dentales: revisón integradora

Recebido: 18/01/2021 | Revisado: 21/01/2021 | Aceito: 03/02/2021 | Publicado: 09/02/2021

Bianca Maria de Souza Cabral

ORCID: https://orcid.org/0000-0001-5622-3949

Universidade de Pernambuco, Brasil

E-mail: bianca.cabral@upe.br

Anna Beathriz Cariolano Marques

ORCID: https://orcid.org/0000-0002-9406-7454

Universidade de Pernambuco, Brasil

E-mail: anna.marques@upe.br

Maria Regina Almeida de Menezes

ORCID: http://orcid.org/0000-0003-3012-3979

Universidade de Pernambuco, Brasil

E-mail: regina.menezes@upe.br

Esdras Gabriel Alves-Silva

ORCID: http://orcid.org/0000-0002-2309-1115

Universidade Estadual de Campinas, Brasil

E-mail: esdras0702@yahoo.com.br

Renata Araújo Gomes de Sá

ORCID: https://orcid.org/0000-0001-6550-8225

Universidade Estadual de Campinas, Brasi

E-mail: renata.sa@upe.br

Eloiza Leonardo de Melo

ORCID: https://orcid.org/0000-0001-5845-1478

Universidade de Pernambuco, Brasil

E-mail: eloiza.leonardo@upe.br

Marleny Elizabeth Márquez de Martínez Gerbi

Universidade de Pernambuco, Brasil

ORCID: https://orcid.org/0000-0001-9174-2541

E-mail: marleny.gerbi@upe.br

Mávio Eduardo Azevedo Bispo

ORCID: https://orcid.org/0000-0002-3781-0272

Universidade de Pernambuco, Brasil

E-mail: meb@ecomp.upe.br

\begin{abstract}
Resumo
A Inteligência Artificial (IA) é um mecanismo proveniente de softwares, que tem como objetivo mimetizar funções cognitivas de seres humanos. Atualmente, a busca por seu uso cresce aceleradamente no ramo da saúde, contemplando diversas áreas, incluindo a Odontologia. A cárie dentária é uma doença dinâmica, multifatorial e mediada por biofilme, que resulta na desmineralização e remineralização fásicas dos tecidos dentários. A cárie dentária é uma das doenças crônicas que mais acometem pessoas ao redor do mundo. O objetivo desse trabalho foi realizar uma revisão integrativa da literatura atual sobre IA na identificação de cárie, acentuando seus benefícios, limitações, relevância e impacto. Com isso, a pergunta norteadora é: o quão viável pode ser o uso de uma tecnologia mais avançada como a IA para o diagnóstico de cáries? A pesquisa foi iniciada por meio de uma busca nos artigos científicos publicados na base de dados eletrônica PubMed, Web of Science, Scopus e Cochrane, utilizando os descritores: "dental caries", "oral health, diagnosis" e "artificial intelligence" indexados no período de 2009 a 2020. Após os critérios de elegibilidade, foram analisados 10 artigos publicados em língua inglesa, portuguesa ou espanhola. A presente revisão integrativa foi capaz de reunir estudos recentes que acentuam o efeito dos métodos atuais da inteligência artificial na saúde bucal, mostrando o seu auxílio à função do cirurgião-dentista, fazendo com que o diagnóstico seja realizado com mais qualidade, precisão e facilidade, obtendo assim uma eficácia maior no tratamento.
\end{abstract}

Palavras-chave: Cárie dentária; Saúde bucal; Diagnóstico; Inteligência artificial. 


\begin{abstract}
Artificial Intelligence (AI) is a software-derived mechanism that aims to mimetize human beings cognitive functions. Currently, the search for its use grows rapidly in the health sector, covering several areas, including dentistry. Dental caries is a dynamic, multifactorial and biofilm-mediated disease that results in phasic demineralization and remineralization of dental tissues. Dental caries is one of the chronic diseases that most affect people around the world. The goal of this article is to perform an integrative review of the current literature on AI in the identification of caries, emphasizing its benefits, limitations, relevance and impact. Thus, the guiding question is: how feasible can the use of a more advanced technology such as AI for the diagnosis of cavities? The research was initiated through a search in the electronic database PubMed, Web of Science, Scopus and Cochrane, using the descriptors: "dental caries", "oral health, diagnosis" and "artificial intelligence" indexed in the period from 2009 to 2020. After the eligibility criteria, 10 articles published in English, Portuguese or Spanish were analyzed. This integrative review was able to gather recent studies that accentuate the effect of current artificial intelligence methods on oral health, showing its aid to a dentist's work, enhancing the diagnosis's quality, precision and ease, thus obtaining greater efficacy in the treatment.
\end{abstract}

Keywords: Dental caries; Oral health; Diagnosis; Artificial intelligence.

\title{
Resumen
}

La inteligencia artificial (IA) es un mecanismo proveniente de softwares, que tiene como objetivo mimetizar las funciones cognitivas de los seres humanos. Actualmente, la búsqueda por su utilización crece aceleradamente en el ramo de la salud, contemplando diversas áreas, incluyendo la Odontología. La carie dental es una enfermedad dinámica, multifactorial y mediada por biopelícula, que resultado en la desmineralización y remineralización física de los tejidos dentales. La carie dental es una de las enfermedades crónicas que más afectan a las personas alredor del mundo. El objetivo de este trabajo fue realizar una revisión integral de la literatura actual sobre IA en la identificación de la carie, acentuando sus beneficios, limitaciones, relevancia e impacto. Por tanto, la pregunta norteadora es: ¿Cuán viable puede ser el uso de una tecnología más avanzada como IA para el diagnóstico de la carie dental? La investigación fue iniciada por medio de una búsqueda de artículos científicos publicados en la base de datos electrónica PubMed, Web of Science, Scopus y Cochrane, utilizando los descriptores: "caries dental", "salud bucal, diagnóstico" y "inteligencia artificial" indexados en el período de 2009 a 2020. Posteriormente a los criterios de elegibilidad, fueran analizados 10 artículos publicados en lengua inglesa, portuguesa o española. La presente revisión integral fue capaz de reunir estudios recientes que acentúan el efecto de los métodos actuales de la inteligencia artificial en la salud bucal, mostrando su auxilio a la función del dentista cirujano, haciendo con que el diagnóstico sea realizado con más calidad, precisión y facilidad. Obteniendo de etsa manera una mayor eficacia en el tratamiento.

Palabras clave: Caries dental; Salud bucal; Diagnóstico; Inteligencia artificial.

\section{Introdução}

A Inteligência Artificial é um ramo da ciência e da informática que foi iniciado com a ideia de desenvolver máquinas para realizarem atividades humanas de maneira autônoma, simulando a inteligência humana. Com vários tipos de tecnologia, estes computadores podem ser treinados para realizarem atividades ao processar uma quantidade específica de dados e reconhecer padrões. Atualmente, as principais tecnologias existentes neste meio são: Machine Learning, Deep Learning ou Processamento de Linguagem Natural (PLN).

O desenvolvimento progressivo da IA em odontologia beneficiará dentistas e pesquisadores para integrar diferentes campos do conhecimento e melhorar o atendimento ao paciente. No entanto, é essencial estar ciente dos erros potenciais na interpretação dos dados por meio de programas de IA. Para minimizar os erros de saída, parece lógico atualmente combinar a tecnologia de IA com metodologias convencionais (Chen, et al., 2020).

A detecção e o diagnóstico rápidos e precisos são fatores importantes na implementação de prevenção e tratamento adequados em pacientes com cáries dentárias. A cárie dentária é uma doença infecciosa bastante comum, caracterizada pela destruição gradual dos tecidos calcificados do dente. Quando a cárie dentária não é diagnosticada corretamente, a lesão pode invadir progressivamente o esmalte, dentina e até mesmo o tecido pulpar, induzindo dor intensa e, por fim, levando à perda da função dentária (J.-H. Lee, et al., 2018). Por isso, tendo em vista que, o quanto antes diagnosticado a doença, mais rapidamente pode-se iniciar o tratamento e, se for realizado de forma eficaz em sua fase inicial, pode ter seu quadro revertido de forma satisfatória, é de extrema importância que as formas de prevenção e diagnóstico sejam cada vez mais estudadas e aprimoradas. 
Desta maneira, o estudo visa apresentar uma revisão integrativa com o objetivo de apurar o uso da Inteligência Artificial na identificação precoce da cárie bucal e, com isso, constatar os seus benefícios, limitações, impacto e relevância para a saúde bucal e para sociedade.

\section{Metodologia}

\subsection{Estratégia de busca}

Este artigo trata-se de uma revisão integrativa da literatura, com metodologia de natureza quantitativa, que foi desenvolvido por um estudo mais aprofundado de artigos encontrados nas bases de dados eletrônicos PubMed, Web of Science, Scopus ou Cochrane, utilizando os descritores "dental caries", "oral health", "diagnosis" e "artificial intelligence", combinado com o período específico para ter o resultado, como mostrado nas Figuras 1, 2, 3 e 4.

Figura 1. Fluxograma da estratégia de busca no PubMed.

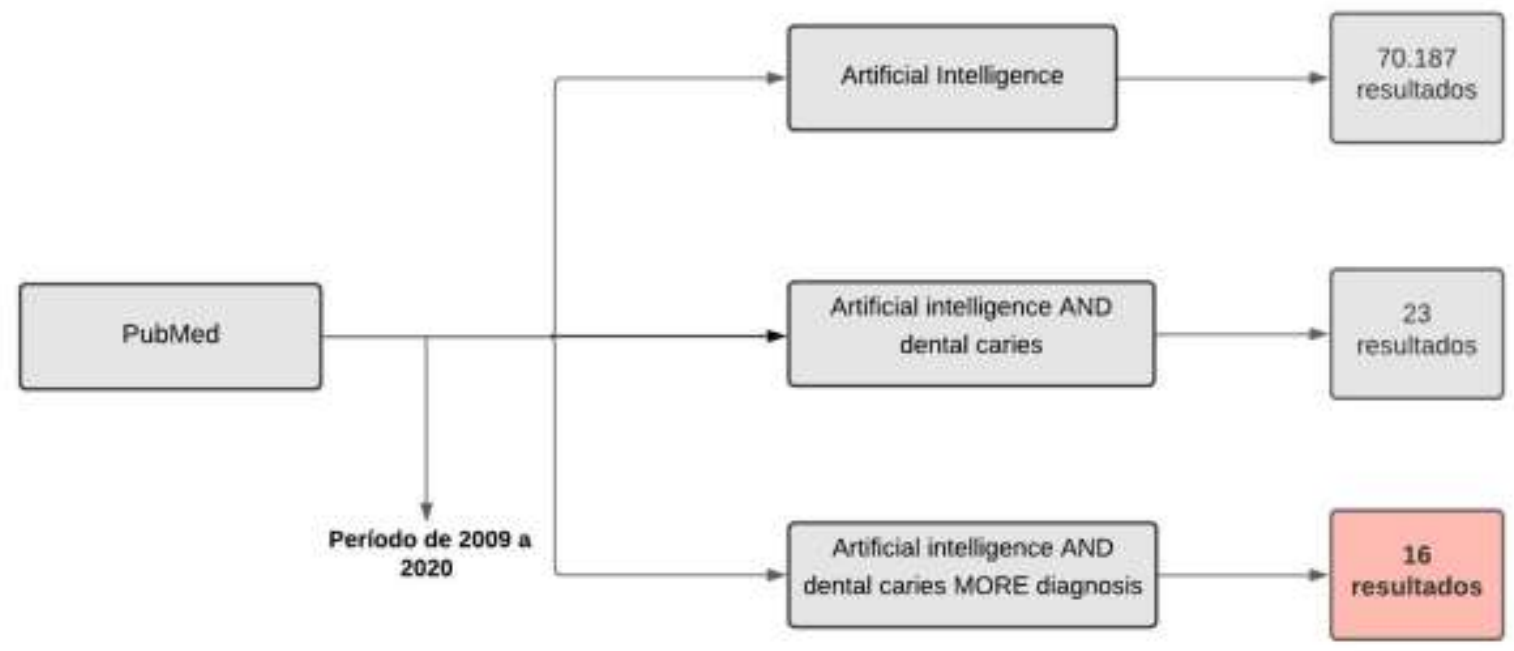

Fonte: Anna Marques e Bianca Cabral (2021).

A partir da busca de artigos científicos no site PubMed, no período de 2009 a 2020, utilizando o descritor "artificial intelligence" obtivemos mais de 70.000 artigos no total. Dessa forma, para focar no nosso objetivo final, acrescentamos o descritor "dental caries" e obtivemos um total de 23 resultados, que continham artigos com o tema mais específico da nossa pesquisa. Para finalizar e termos um resultado ainda mais claro, adicionamos aos descritores anteriores, "diagnosis" e obtivemos 16 artigos. 
Figura 2. Fluxograma da estratégia de busca no Cochrane.

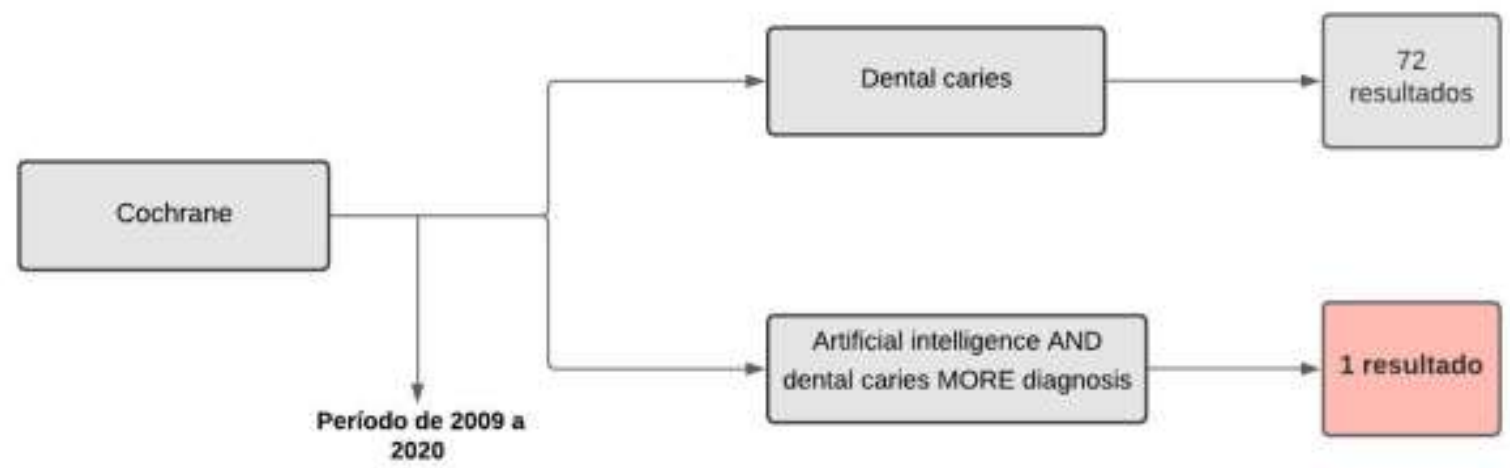

Fonte: Anna Marques e Bianca Cabral (2020).

No site Cochrane pesquisamos primeiramente o descritor "dental caries" e obtivemos 72 resultados, acrescentamos o descritor "artificial intelligence" para que chegássemos ao objetivo da pesquisa e o resultado foi 1 artigo, acrescentando o descritor "diagnosis" nenhum artigo foi encontrado.

Figura 3. Fluxograma da estratégia de busca no Scopus.

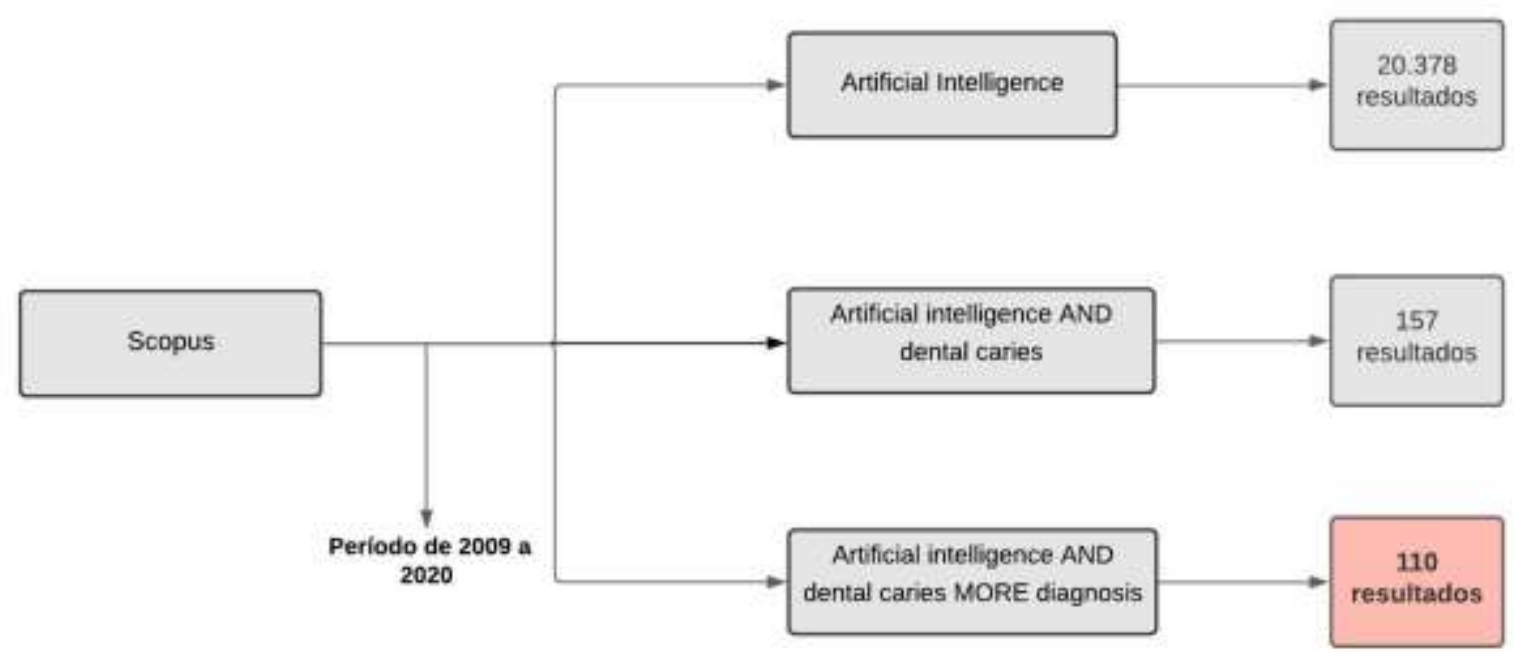

Fonte: Anna Marques e Bianca Cabral (2021).

No Scopus, inicialmente utilizamos o descritor "dental caries" e obtivemos mais de 20.000 resultados, afinando a pesquisa acrescentamos "artificial intelligence" e o resultado foi mais de 150 artigos, para finalizar, foi incluído o descritor "diagnosis" e conseguimos obter 110 resultados no total. 
Figura 4. Fluxograma da estratégia de busca no Web of Science.

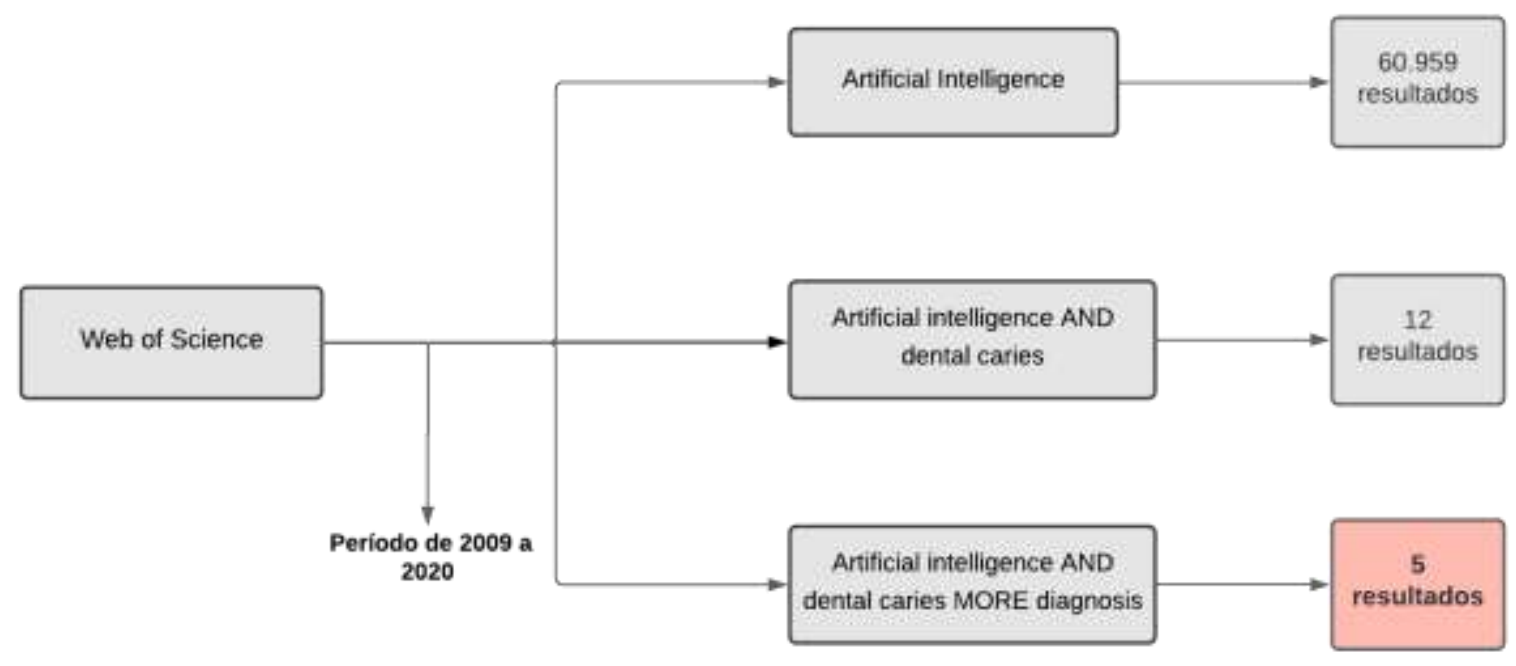

Fonte: Anna Marques e Bianca Cabral (2021).

Já no Web of Science, com o primeiro descritor obtivemos mais de 60.000 resultados, afinando a pesquisa com "dental caries" conseguimos chegar aos 12 e acrescentando "diagnosis", o resultado final foram 5 artigos.

\subsection{Seleção das publicações e extração de dados}

A avaliação e seleção de artigos foi efetuada por dois pesquisadores, de modo autônomo, com exposição dos resultados e diálogo sobre o que se foi lido. Então, a seleção das publicações foi separada em duas partes: (1) leitura dos resumos e (2) discussão dos textos selecionados.

\subsection{Critérios de elegibilidade dos artigos}

Para as fontes, foram consideradas como critérios de inclusão artigos originais disponibilizados na íntegra e revisões de literatura, publicados em língua inglesa ou portuguesa nos períodos de 2009 a 2020, que tratavam de estudos e pesquisas sobre a Inteligência Artificial associada à Odontologia e especificamente ao tratamento de cáries dentárias. A seguir, a Figura 5 que ilustra a estratégia de busca de acordocom os critérios citados. 
Figura 5. Fluxograma de estratégia de busca e seleção dos artigos.

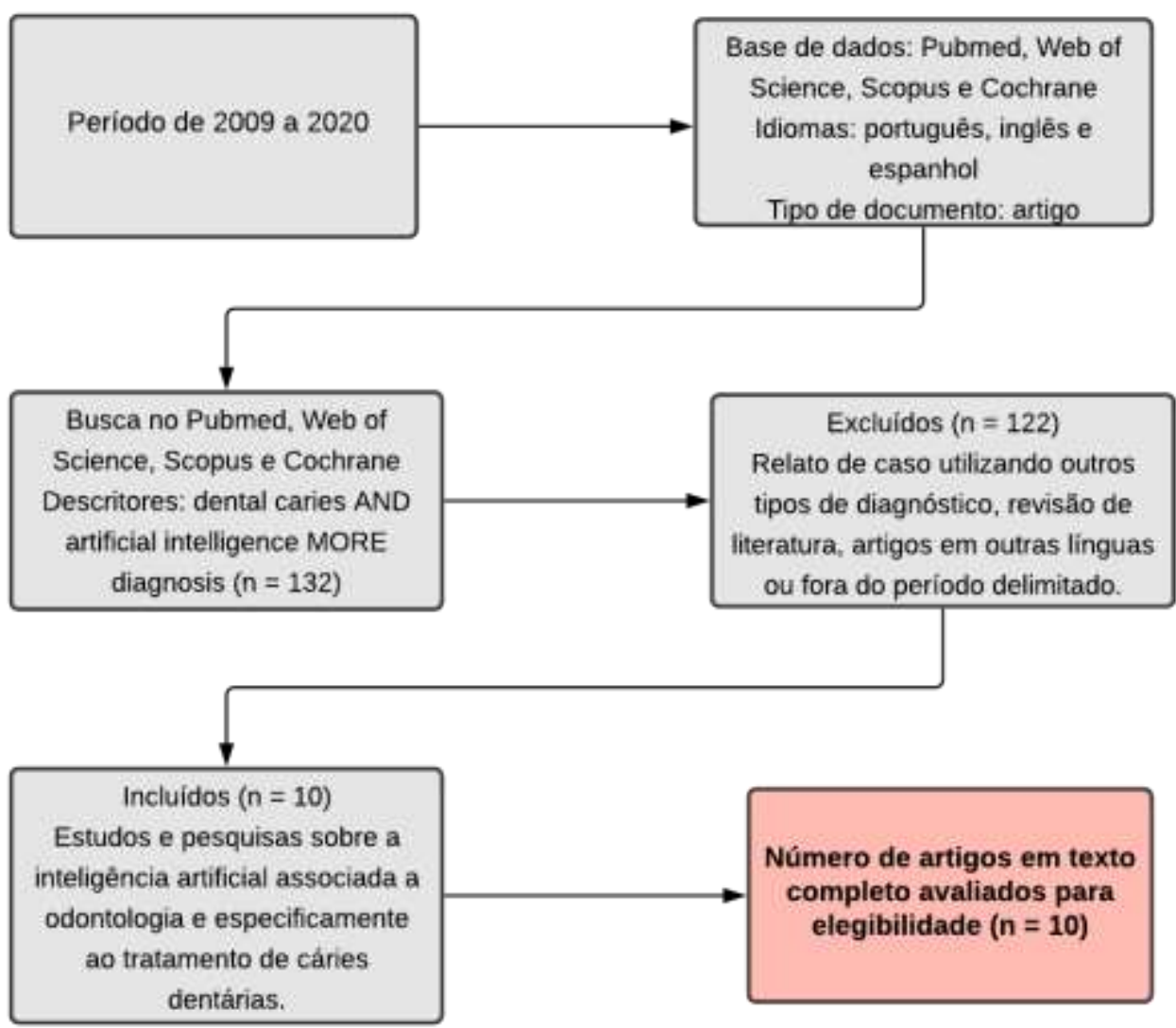

Fonte: Anna Maques e Bianca Cabral (2021).

Esta metodologia seguiu as recomendações PRISMA (Principais Itens para Relatar Revisões Sistemáticas e Metanálise). Os artigos que não se encaixavam nos critérios de inclusão, ou repetidos, foram excluídos. Posteriormente, dos artigos restantes, foi realizada a leitura completa, chegando a uma amostra final de 10 artigos incluídos nesta revisão.

Não foram utilizadas teses, dissertações e monografias, visto que a realização de uma busca desses estudos é inviável logisticamente e a base de dados utilizada não tem esse tipo de publicação.

\section{Resultados}

Essa revisão integrativa encontrou estudos recentes que abordam os avanços da Inteligência Artificial na área da saúde bucal, com foco nos benefícios, limitações, relevância e impacto no que diz respeito a detecção preoce da lesão cariosa como mostra no Quadro 1. 
Quadro 1. Seleção dos artigos de acordo com o autor, tipo de estudo, objetivos, metodologia, resultados e conclusão.

\begin{tabular}{|c|c|c|c|c|}
\hline $\begin{array}{l}\text { AUTOR E } \\
\text { ESTUDO }\end{array}$ & OBJETIVO & METODOLOGIA & RESULTADOS & CONCLUSÃO \\
\hline $\begin{array}{l}\text { Chen YW. et } \\
\text { al.(2020) }\end{array}$ & $\begin{array}{l}\text { Avaliar e comparar a } \\
\text { progressão do uso da } \\
\text { Inteligência Artificial } \\
\text { no diagnóstico } \\
\text { odontológico. }\end{array}$ & $\begin{array}{l}\text { Pesquisas eletrônicas foram exploradas. Os descritores } \\
\text { usados foram: "artificial intelligence", "AI", "machine } \\
\text { learning" e "deep learning". Os descritores "destistry", } \\
\text { "dental" e "dental care", foram usados como termos de } \\
\text { pesquisa adjacentes. }\end{array}$ & $\begin{array}{l}\text { Uma rede de deep learning } \\
\text { pré-treinada } \\
\text { (GoogleNetInception v3) } \\
\text { pode ser usada para o } \\
\text { diagnóstico de cárie dentária, } \\
\text { com a precisão de } \\
\text { identificação em pré-molares, } \\
\text { molares e entre pré-molares e } \\
\text { molares em } 89 \%, 88 \% \text { e } 82 \% \text {, } \\
\text { respectivamente. }\end{array}$ & $\begin{array}{l}\text { A radiografia baseada em } \\
\text { IA mostra um grande } \\
\text { potencial de auxiliar os } \\
\text { dentistas à realização de } \\
\text { um diagnóstico comum a } \\
\text { referência adicional. }\end{array}$ \\
\hline $\begin{array}{l}\text { Hung M. et al. } \\
(2019)\end{array}$ & $\begin{array}{lr}\text { Avaliar a utilização do } \\
\text { método } & \text { machine } \\
\text { learning } & \text { em } \\
\text { inteligência } & \text { artificial } \\
\text { para detecção de cárie } \\
\text { radicular, medindo o } \\
\text { desempenho } \\
\text { modelo. }\end{array}$ & $\begin{array}{l}\text { Foram usados diversos métodos de machine learning, } \\
\text { para construir uma ferramenta capaz de classificar } \\
\text { variáveis quanto a presença ou ausência de cárie } \\
\text { radicular. Foram computados a precisão, } \\
\text { sensibilidade, especificidade e área sob a curva de } \\
\text { operação do receptor. }\end{array}$ & $\begin{array}{l}\text { No total, } 2.448 \text { imagens } \\
\text { foram anotadas neste estudo. } \\
\text { Os resultados mostram que } \\
\text { para radiografias panorâmicas } \\
\text { usando a tarefa de detecção de } \\
\text { radiolucências, OMF teve em } \\
\text { média } 31 \% \text { dos casos } \\
\text { diagnosticados falsamente } \\
\text { como positivos. OTPR médio } \\
\text { indicou que 49\% de todas as } \\
\text { radiolucências foram } \\
\text { perdidas. A OMF de } \\
\text { desempenho foi } \\
\text { significativamente menor em } \\
\text { termos de TPR em } \\
\text { comparação com PPV com } \\
\text { base em um teste de postos } \\
\text { sinalizados de Wilcoxon (p = } \\
0,003 \text { ). }\end{array}$ & $\begin{array}{l}\text { A utilização deste método } \\
\text { teve um bom desempenho } \\
\text { e os algoritimos da } \\
\text { machine learning } \\
\text { permitem a execução e } \\
\text { utilização clínica por } \\
\text { profissionais } \\
\text { odontológicos ou não, que } \\
\text { estejam qualificados. }\end{array}$ \\
\hline
\end{tabular}




\begin{tabular}{|c|c|c|c|c|}
\hline $\begin{array}{l}\text { Jae-Hong Lee et } \\
\text { al. (2018) }\end{array}$ & $\begin{array}{lr}\text { Avaliar a eficácia de } \\
\text { algoritimos de } & \text { Rede } \\
\text { Neural Convolucional } \\
\text { profunda } & \text { (Deep } \\
\text { Convolutional } & \text { Neural } \\
\text { Network) para detecção } \\
\text { e diagnostico de } & \text { cárie } \\
\text { dentária } & \text { em } \\
\text { radiografias } & \\
\text { periapicais. }\end{array}$ & $\begin{array}{l}\text { Foram divididas } 3.000 \text { imagens radiográficas } \\
\text { periapicais em um conjunto de dados de treinamento e } \\
\text { validação (n=2.400) e um conjunto de dados de teste } \\
\text { (n=600). A precisão, diagnóstico, sensibilidade, } \\
\text { especificidade, valor preditivo positivo e negativo, } \\
\text { curva de característica do receptor (ROC) e área sob a } \\
\text { curva (AUC) foram calculados para detecção e } \\
\text { desempenho diagnóstico do algoritmo deep CNN. }\end{array}$ & $\begin{array}{l}\text { A precisão diagnóstica de pré- } \\
\text { molares, molares e modelos } \\
\text { de pré-modelares e molares } \\
\text { foram } 89,0 \%, 88,0 \% \text {, e } \\
82,0 \% \text {, respectivamente. O } \\
\text { algoritimo CNN alcançou } \\
\text { uma área sob a curva (AUC) } \\
\text { de } 0,917 \text { no pré-molar, } 0,890 \\
\text { no molar e } 0,845 \text { em ambos } \\
\text { modelos pré-molares e } \\
\text { molares. O modelo pré-molar } \\
\text { foi o que obteve a melhor } \\
\text { AUC. }\end{array}$ & $\begin{array}{l}\text { Este estudo forneceu um } \\
\text { desempenho } \\
\text { consideravelmente bom na } \\
\text { detecção de cárie dentária } \\
\text { em radiografias } \\
\text { periapicais. }\end{array}$ \\
\hline $\begin{array}{l}\text { Oleksandr A. U. } \\
\text { et al.(2020) }\end{array}$ & 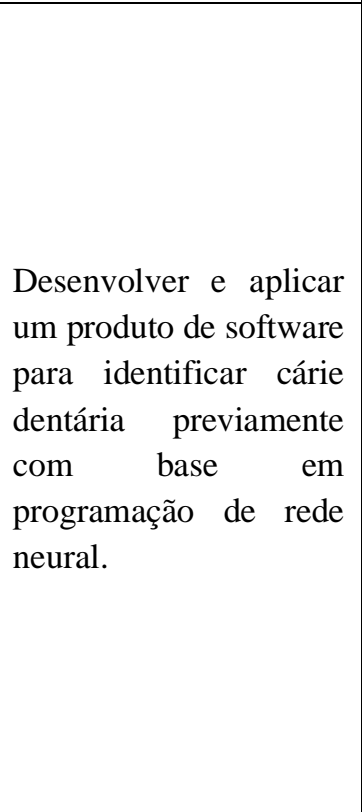 & $\begin{array}{l}\text { Exame dentário dividido em três grupos; } 26 \text { crianças } \\
\text { de 6-7 anos de idade; } 34 \text { de } 12-15 \text { anos e } 13 \text { adultos de } \\
35-44 \text { anos. Os resultados de cada exame foram } \\
\text { utilizados como base para treinamento e construção da } \\
\text { rede neural. O índice de saída foi determinado pelo } \\
\text { aumento da intesidade da cárie, levando em conta o } \\
\text { número de cavidades encontrados no exame. Para } \\
\text { construir a rede neural, foi utilizado um programa de } \\
\text { linguagem Python. Os produtos de software utilizados } \\
\text { foram: Pandas, Tensor Flow, Keras, Scikit-learn, } \\
\text { Matplotlib e Jupyter Notebook. }\end{array}$ & $\begin{array}{l}\text { Foi observado que a } \\
\text { intesidade de cárie dentária } \\
\text { em crianças de } 6-7 \text { anos é de } \\
3.85 \pm 0.27 \text {. Nas crianças de } \\
12-15 \text { foi de } 2.15 \pm 0.24 \text {. Já } \\
\text { nos adultos de } 33-44 \text { anos, } \\
6.69 \pm 0.38 \text {, e foi o nível mais } \\
\text { alto. Os resultados obtidos } \\
\text { foram utilizados para treinar a } \\
\text { rede neural e depois foi obtido } \\
\text { um resultado com base no } \\
\text { cálculo do índice precisão de } \\
\text { trabalho da rede neural, que } \\
\text { mostrava } 61 \text { previsões } \\
\text { verdadeiras e 12 falsas } \\
\text { (precisão de } 83,56 \% \text { ). Devido } \\
\text { à eficácia, foi criada uma } \\
\text { interface gráfica de usuário } \\
\text { para o aplicativo de software } \\
\text { "CariesPro". }\end{array}$ & $\begin{array}{l}\text { A rede neural resultante e } \\
\text { o produto de software } \\
\text { obteve um resultado } \\
\text { positivo de } 83,56 \% \text { e com } \\
\text { isto, se utilizado, poderá } \\
\text { prever cáries dentárias } \\
\text { mais facilmente na prática } \\
\text { clínica diária. }\end{array}$ \\
\hline $\begin{array}{l}\text { F. Casalegno et } \\
\text { al. (2019) }\end{array}$ & $\begin{array}{l}\text { Apresentar um modelo } \\
\text { de deep learning para a }\end{array}$ & $\begin{array}{l}\text { Treinamento de um modelo baseado em CNN para a } \\
\text { detecção e classificação de imagens de lesões } \\
\text { dentárias obtidas com imagens de TI no infravermelho }\end{array}$ & $\begin{array}{l}\text { Os resultados indicam que o } \\
\text { modelo de linha de base deu } \\
\text { um mIOU (média de }\end{array}$ & $\begin{array}{l}\text { Neste trabalho, foi } \\
\text { demonstrado o uso de uma }\end{array}$ \\
\hline
\end{tabular}




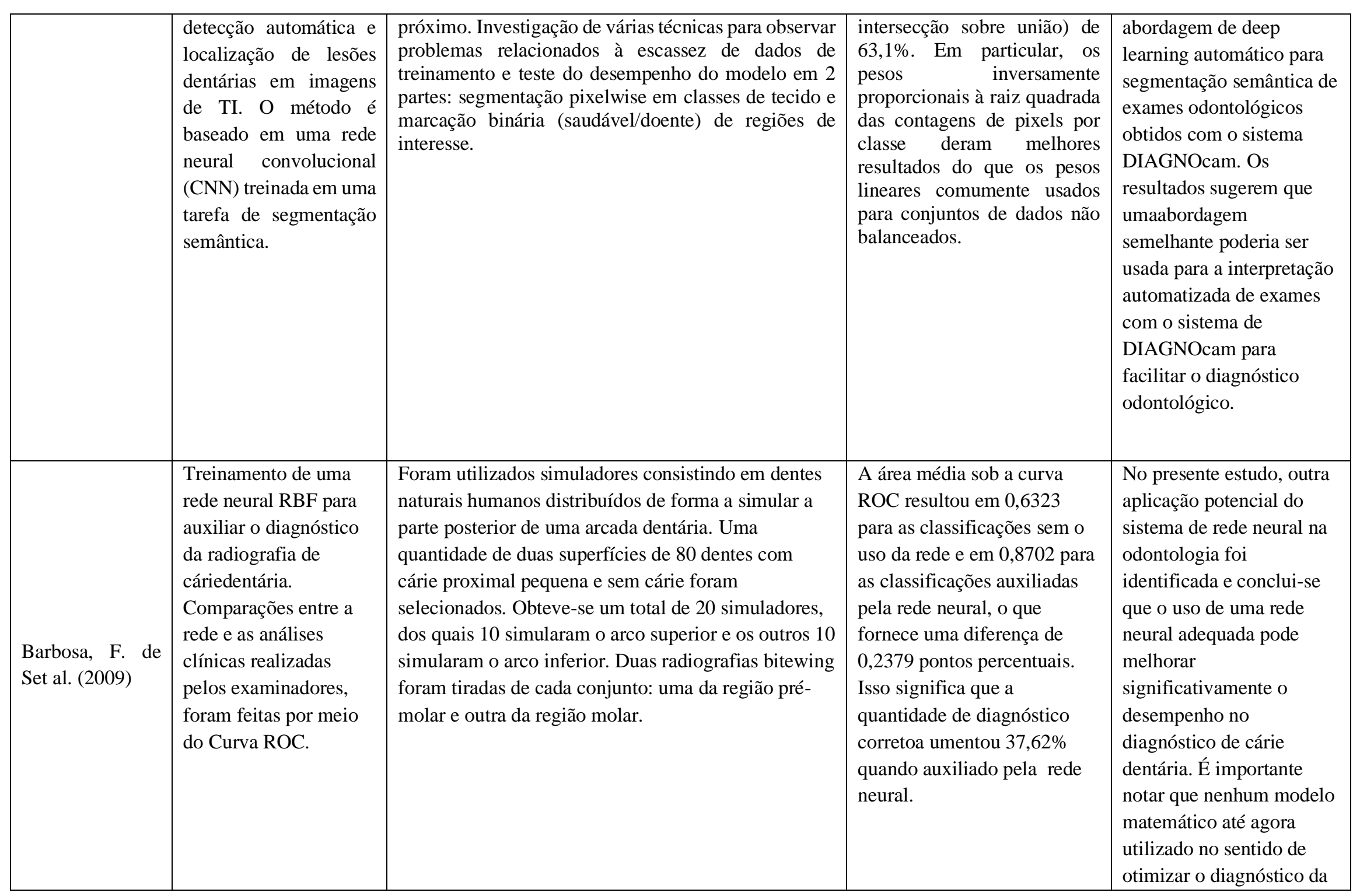




\begin{tabular}{|c|c|c|c|c|}
\hline & & & & $\begin{array}{l}\text { cárie dentária se mostrou } \\
\text { suficientemente preciso ou } \\
\text { completo, devido à } \\
\text { complexidade biológica } \\
\text { destadoença. }\end{array}$ \\
\hline $\begin{array}{l}\text { Charvát, J. et al. } \\
\text { (2020) }\end{array}$ & $\begin{array}{l}\text { O trabalho propõe o } \\
\text { uso de métodos de } \\
\text { inteligência } \\
\text { computacional para o } \\
\text { processamento de } \\
\text { dados e da qualidade } \\
\text { do tecido dentário } \\
\text { usando a medição para } \\
\text { ajudar na estimativa do } \\
\text { diagnóstico. }\end{array}$ & $\begin{array}{l}\text { Todos os conjuntos de dados foram registrados pelo } \\
\text { sistema espectrométrico que usou uma fonte de luz } \\
\text { alógena de banda larga e uma sonda portátil. A fibra } \\
\text { de coleta foi conectada a um espectrômetro comum } \\
\text { detector InGaAs. O critério de classificação da } \\
\text { entrada foi criado com base no Sistema Internacional } \\
\text { de Detecção e Avaliação de Cáries (ICDAS). Este } \\
\text { sistema de pontuação [21,22] foi usado para } 578 \\
\text { tecidos e simplificado por um dentista experiente. Na } \\
\text { fase inicial, duas categorias foram analisadas para } \\
\text { distinguir tecidos saudáveis (classe 1) e não } \\
\text { saudáveis (classe 2). }\end{array}$ & $\begin{array}{l}\text { Os tecidos afetados diferem } \\
\text { dos tecidos saudáveis pelo } \\
\text { maior conteúdo de água. Este } \\
\text { efeito é visível para o valor } \\
\text { máximo de refletividade } \\
\text { associado a comprimentos de } \\
\text { onda maiores que } 800 \mathrm{~nm} \text {. Os } \\
\text { tecidos saudáveis têm, além } \\
\text { disso, menor desvio padrão } \\
\text { dos valores das } \\
\text { características e formam } \\
\text { aglomerados mais compactos } \\
\text { do que os tecidos com cárie. }\end{array}$ & $\begin{array}{l}\text { Os resultados obtidos } \\
\text { sugerem que o método } \\
\text { proposto é capaz de } \\
\text { classificar experimentos } \\
\text { com a precisão suficiente } \\
\text { para distinguir classes de } \\
\text { tecidos saudáveis e } \\
\text { nãosaudáveis. O algoritmo } \\
\text { resultante inclui a } \\
\text { metodologia de seleção de } \\
\text { recursos com base em } \\
\text { suas propriedades } \\
\text { estatísticas. }\end{array}$ \\
\hline You et al.(2020) & $\begin{array}{l}\text { Estudo pioneiro que } \\
\text { usa redes para detectar } \\
\text { placa dentária com } \\
\text { base em um conjunto } \\
\text { de dados de fotos de } \\
\text { dentes decíduos. Além } \\
\text { disso, avaliam o } \\
\text { diagnóstico de } \\
\text { desempenho de um } \\
\text { sistema de IA que usa } \\
\text { deep learning para } \\
\text { detectar placa dentária }\end{array}$ & $\begin{array}{l}\text { Uma CNN foi adotada e } 886 \text { fotos intraorais de } \\
\text { dentes decíduos foram usados para treinamento. Um } \\
\text { dentista pediatra examinou as fotos e marcou as } \\
\text { regiões contendo placa. A média de intersecção sobre } \\
\text { união métrica (MIoU) foi empregada para indicar a } \\
\text { precisão da detecção. }\end{array}$ & $\begin{array}{l}\text { O MIoU para detecção de } \\
\text { placa nas fotos de dentes } \\
\text { testados foi de } 0,726 \pm 0,165 \text {. } \\
\text { O MIoU do dentista foi de } \\
0,695 \pm 0,269 \text { quando } \\
\text { diagnosticou pela primeira } \\
\text { vez as } 98 \text { fotos tiradas pela } \\
\text { câmera digital e } 0,689 \pm 0,253 \\
\text { após } 1 \text { semana. Em } \\
\text { comparação com o dentista, o } \\
\text { modelo AI demonstrou um } \\
\text { MIoU superior }(0,736 \pm \\
0,174) \text { e o resultado não }\end{array}$ & $\begin{array}{l}\text { O modelo de IA mostrou } \\
\text { um desempenho } \\
\text { clinicamente aceitável na } \\
\text { detecção de placa dentária } \\
\text { em dentes decíduos em } \\
\text { comparação com um } \\
\text { dentista pediatra } \\
\text { experiente. }\end{array}$ \\
\hline
\end{tabular}


Research, Society and Development, v. 10, n. 2, e18310212117, 2021

(CC BY 4.0) | ISSN 2525-3409 | DOI: http://dx.doi.org/10.33448/rsd-v10i2.12117

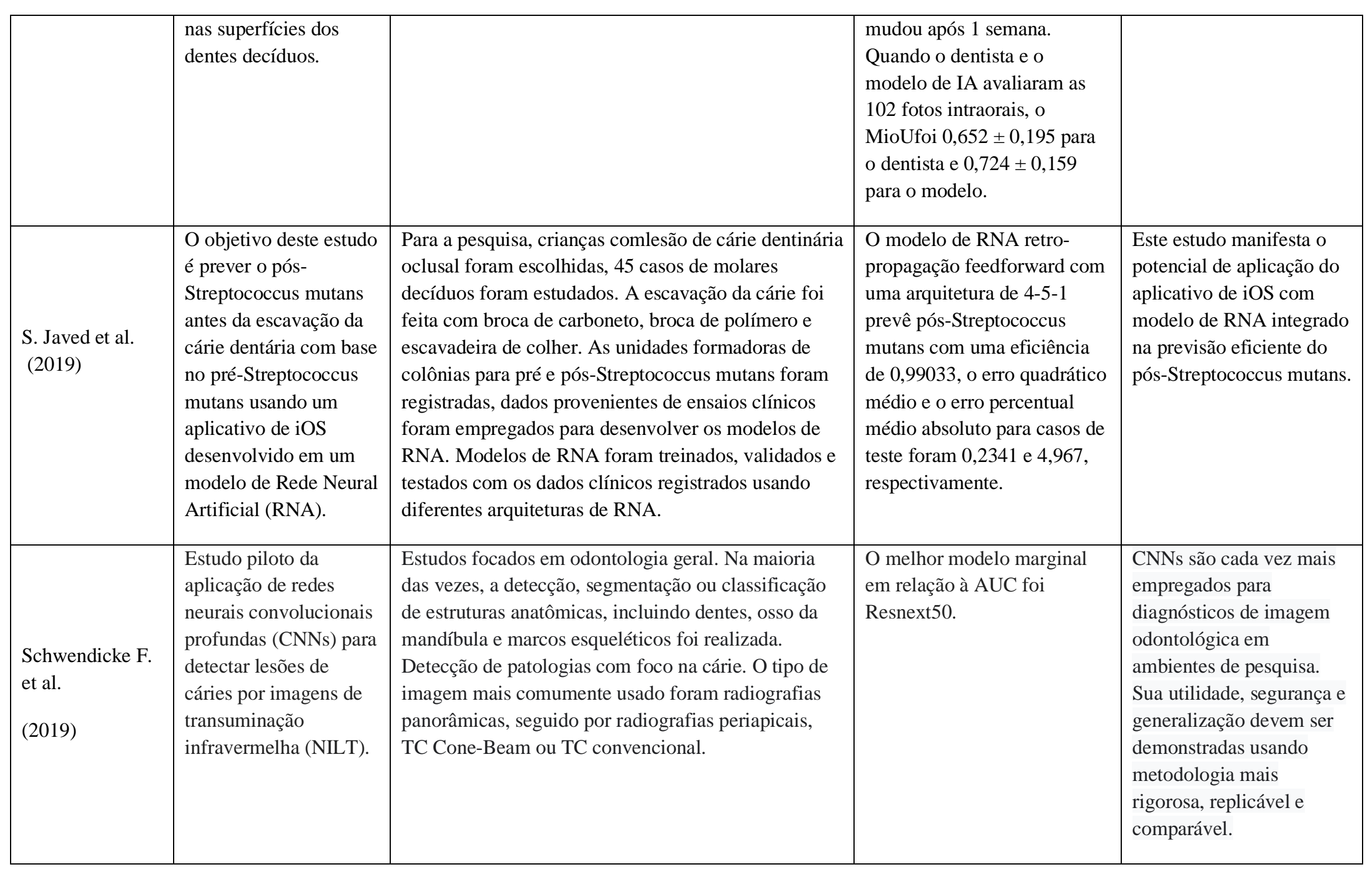

Fonte: Anna Marques e Bianca Cabral (2021). 


\subsection{Tipos e quantidades de artigos}

Foram selecionados dentre os critérios, 10 artigos científicos que contivessem exames ou pesquisas. Os resultados mostraram que o emprego da Inteligência Artificial vem evoluindo com técnicas eficazes que mostram o quão significativo está sendo o avanço tecnológico para a área de saúde com o intuito de ampliar, nesse caso, o diagnóstico precoce de doenças como a cárie. Foram observadas técnicas como aplicação de redes neurais convolucionais profundas e transuminação infravermelha, porém ainda é um tópico em andamento conforme o quadro 1, necessitando de um maior número de pesquisas científicas e clínicas, com um maior número de pacientes e aplicações para se obter conclusões mais significantes.

\section{Discussão}

O bom desempenho da Inteligência Artificial na identificação de cáries é evidenciado na literatura. A utilização do Deep Learning vem tomando grande espaço nesse âmbito, em especial as Redes Neurais Convolucionais (CNNS), que, como salienta Schwendickeet al. (2019), é uma ramificação da IA e tem grande potencial para detecção e diagnóstico de cárie dentária, pois fornece um desempenho consideravelmente bom na detecção em radiografias periapicais.

Juntamente com o infravermelho, a CNN demonstra uma capacidade discriminatória satisfatória para localizar lesões de cáries em material de imagem NILT. Com isso, Schwendicke et al. (2019) sugere que as CNNs se mostram úteis para auxiliar na detecção de cárie baseada em imagens de transluminação infravermelha, em vários âmbitos de práticas dentais e conFigurações odontológicas não-convencionais.

Segundo You et al. (2020), esse modelo demonstra um desempenho clinicamente aceitável nadetecção de placa dentária em dentes decíduos, contemplando a odontopediatria. Outros procedimentos podem ser derivados do Deep Learning, como apresenta F. Casalegno et al. (2019), sobre o uso de uma abordagem de deep learning automático para segmentação semântica de exames odontológicos obtidos com o sistema DIAGNOcam.

Por tratar-se de uma tecnologia recente na área da saúde, traz consigo implicações principalmente pela pequena quantidade de estudos feitos. Porém, cada vez mais vem se destacando o interesse nessa área de pesquisa. A radiografia baseada em IA mostra um grande potencial de auxiliar os dentistas na realização de um diagnóstico com uma referência adicional. De acordo com Chen Yw. et al. (2020), acredita-se que um novo tipo de abordagem interprofissional entre dentistas, pesquisadores e engenheiros serão essenciais para o desenvolvimento dessa ferramenta. Como tudo que se correlaciona à saúde compromete a sociedade, as pesquisas e investimento em aplicação das redes neurais na identificação de cárie dental se mostram essenciais para a melhoria e promoção da saúde da população, incluindo o ramo da saúde pública.

O presente trabalho trata-se de uma revisão integrativa da literatura, nele foram analisados estudos relevantes sobre as diversas redes neurais relacionadas à Inteligência Artificial. Foi realizada uma síntese de conhecimentos relacionados a esse assunto, acentuando seus benefícios, limitações, relevância e impacto. Este estudo se torna relevante, pois encontramos 5 artigos sobre Inteligência Artificial na identificação de cárie dental relacionada ao seu diagnóstico e outros 12 artigos que tratam de uma visão menos específica dessa utilização de inteligência na odontologia.

Ao colocarmos os descritores "Dental caries", "Artificial Intelligence", e "Diagnosis", encontramos trabalhos que não eram compatíveis com nosso objetivo, o que comprova a importância da realização de estudos sobre esse tema, para fortalecer as técnicas da utilização dos sistemas, juntamente com o algoritmo CNNS, na odontologia. Por sua vez, ao usarmos os bancos de dados, nos deparamos com a dificuldade de encontrar estudos relativos a esse tema, então a solução foi refinar a pesquisa até chegarmos aos artigos que se enquadravam no nosso objetivo. Observamos que foi fundamental acrescentar "and" e "more" para afinar e obtermos tais resultados. As plataformas usadas foram Pubmed, Cochrane, Web of Science e Scopus, que são referências 
mundiais. Dessa forma, nossos resultados contêm fontes seguras e uso de descritores cadastrados em banco de dados e plataformas com credibilidade acadêmica.

Levando-se em consideração esses aspectos, é notável que a implementação da Inteligência Artificial na odontologia trará grandes frutos, como ganho de performance e produtividade dos profissionais. Segundo Hung M. et al. (2020), a implantação desse método é importante para que se obtenha uma intervenção e tratamento precoce da cárie radicular, por isso, os profissionais devem ser encorajados adotá-lo, e assim, terão uma medicina dental de precisão.

A relevância desta pesquisa se dá pela importância do estudo de novos recursos emergentes, como é o caso da Inteligência Artificial no ramo da saúde. Isso implica um tratamento mais eficaz em relação a cárie, já que é a doença bucal mais conhecida. O impacto que esta pesquisa nos causou foi aguçar a vontade de explorar novas áreas que possivelmente farão parte do nosso futuro na profissão e ter em mente diferentes tipos de abordagens.

\section{Considerações Finais}

Levando-se em consideração o que foi analisado, podemos notar que o avanço tecnológico na área odontológica vem evoluindo de forma gradativa, e que seu uso tem impacto positivo graças a sua eficácia, praticidade e agilidade, quando comparados a métodos que vêm sendo utilizados há anos na área de saúde. Portanto, cabe aos profissionais dessa área investir, tanto no estudo da Inteligência Artificial para seu aperfeiçoamento, como também em mais treinamento das máquinas para que o resultado seja totalmente satisfatório.

\section{Referências}

Barbosa, Flávio de Souza et al. Using a neural network for supporting radiographic diagnosis of dental caries, Applied Artificial Intelligence:An International Journal, 2009. https://www.tandfonline.com/doi/abs/10.1080/08839510903246757

Casalegno, F et al.Caries Detection with Near-Infrared Transillumination Using Deep Learning. PubMed, 2020. https://pubmed.ncbi.nlm.nih.gov/31449759/

Charvát, J et al. Diffuse reflectance spectroscopy in dental caries detection and classification. Signal, ImageandVideo, 2020. https://doi.org/10.1007/s11760020-01640-4

Chen, Yo-Wei et al. Artificial intelligence in dentistry: current applications and future perspectives. PubMed, 2020. https://pubmed.ncbi.nlm.nih.gov/32020135/

Filho, J. C. B. L et al. Methods for detection of dental caries: from traditional to new technologies for clinical use. PubMed, 2011. http://files.bvs.br/upload/S/1983-5183/2012/v23n3/a2769.pdf

Hung, Man et al. Application of machine learning for diagnostic prediction of root caries. Web of Science, $2019 . \quad$ : https://www.researchgate.net/profile/Man_Hung2/publication/334258703_Application_of_machine_learning_for_diagnostic_prediction_of_root_caries/links/ 5ee4ba8ea6fdcc73be7815ad/Application-of-machine-learning-for-diagnostic-prediction-of-root-caries.pdf

Javed, S. et al. Development of artificial neural network model for prediction of post-streptococcus mutans in dental caries. Scopus, 2019.

https://www.sciencedirect.com/science/article/pii/S0169260719316347

Kositbowornchai, S. et al. An Artificial Neural Network for Detection of Simulated Dental Caries.International Journal of Computer Assisted Radiology and Surgery, 2006. https://doi.org/10.1007/s11548-006-0040-x

Lee, Jae-Hong et al. Detection and diagnosis of dental caries using a deep learning-based convolutional neural network algorithm. Web of Science, 2018. https://www.sciencedirect.com/science/article/abs/pii/S0300571218302252

Orhan, K. et al. Evaluation of artificial intelligence for detecting periapical pathosison cone-beam computed tomography scans. International Endodontic Journal, 2020. https://onlinelibrary.wiley.com/doi/abs/10.1111/iej.13265

Park, WookJoo et al. History and application of artificial neural networks in dentistry. European journal of dentistry, 2018. https://doi.org/10.4103/ejd.ejd_325_18

Pereira A. S. et al. (2018). Metodologia da pesquisa científica. UAB/NTE/UFSM. https://repositorio.ufsm.br/bitstream/handle/1/15824/Lic _Computacao_Metodologia-Pesquisa-Cientifica.pdf?sequence=1

Prados-Privado, M et al. Dental Caries Diagnosis and Detection Using Neural Networks: A Systematic Review. Journal of Clinical Medicine, 2020. https://doi.org/10.3390/jcm9113579 
Research, Society and Development, v. 10, n. 2, e18310212117, 2021

(CC BY 4.0) | ISSN 2525-3409 | DOI: http://dx.doi.org/10.33448/rsd-v10i2.12117

Schwendicke, Falk et al. Convolutional neural networks for dental image diagnostics: A scoping review. Scopus, 2019. https://www.sciencedirect.com/science/article/pii/S0300571219302283

You, $\mathrm{W}$ et al. Deep learning-based dental plaque detection on primary teeth: a comparison with clinical assessments. BMC Oral Health, 2020. https://doi.org/10.1186/s12903-020-01114-6

Yüzbaşığlu, E. Attitudes and perceptions of dental students towards artificial intelligence. Journal of Dental Education, 2020. https://doi.org/10.1002/jdd.12385 